Review Article

\title{
Role of High-Dose Chemotherapy and Autologous Hematopoietic Cell Transplantation for Children and Young Adults with Relapsed Ewing's Sarcoma: A Systematic Review
}

\author{
Pavan Tenneti (D), ${ }^{1}$ Umar Zahid, ${ }^{2}$ Ahmad Iftikhar, ${ }^{3}$ Seongseok Yun $\left({ }^{4},{ }^{4}\right.$ Atif Sohail, ${ }^{3}$ \\ Zabih Warraich, ${ }^{3}$ and Faiz Anwer $\mathbb{1}^{3}$ \\ ${ }^{1}$ Department of Medicine, University of Arizona, Tucson, AZ 85721, USA \\ ${ }^{2}$ Department of Biostatics, Mel and Enid Zuckerman College of Public Health, University of Arizona, \\ Tucson, AZ 85721, USA \\ ${ }^{3}$ Division of Hematology and Oncology, Department of Medicine, University of Arizona, Tucson, AZ 85721, USA \\ ${ }^{4}$ Malignant Hematology, H. Lee Moffitt Cancer Center and Research Institute, Tampa, FL 33612, USA
}

Correspondence should be addressed to Faiz Anwer; anwerf@email.arizona.edu

Received 30 January 2018; Revised 24 April 2018; Accepted 13 May 2018; Published 3 June 2018

Academic Editor: Valerae O. Lewis

Copyright @ 2018 Pavan Tenneti et al. This is an open access article distributed under the Creative Commons Attribution License, which permits unrestricted use, distribution, and reproduction in any medium, provided the original work is properly cited.

Background. Relapsed Ewing's sarcoma (RES) is an aggressive malignancy with poor survival. Although high-dose chemotherapy (HDCT) with autologous stem cell transplantation (ASCT) given after conventional chemotherapy (CC) has shown survival benefits, it is not generally used in the United States for RES. We performed a systemic review to evaluate the benefits of HDCT for RES. Methods. Literature search involved Medline, Embase, and Cochrane database. We included studies with RES patients treated with HDCT/ASCT. Results. Twenty-four studies with total of 345 reported RES patients that got HDCT were included in final analysis. Seventeen studies had patients with multiple malignancies including RES, while seven had only RES patients. At 2 and 3-5 years, event-free survival (EFS) in studies with only RES patients ranged $42-47 \%$ and $20-61 \%$ and overall survival (OS) ranged $50-66 \%$ and $33-77 \%$, respectively. In studies with combined patients that reported outcomes of RES separately, the EFS at $1-3$ and 4 years was $36-66 \%$ and $17-50 \%$, respectively. The OS at $1-2$ and 3-4 years was $40-60 \%$ and $50-70 \%$. Conclusions. Most studies using HDCT/ASCT as consolidation regimen showed improved survival benefits compared to CC. Randomized controlled studies are needed to determine true clinical benefits of HDCT followed by ASCT in patients with RES.

\section{Introduction}

Patients with localized primary Ewing's sarcoma (ES) have 60-70\% 5-year overall survival (OS) with multimodality treatment [1]. In patients with primary metastatic Ewing's sarcoma, the OS rate is $20-40 \%$ with treatment [1]. Approximately $30-40 \%$ of patients with primary Ewing's sarcoma who initially achieved remission after front-line treatment experience disease relapse, and the prognosis in these patients was shown to be dismal [2]. The poor prognostic factors at relapse include relapse time less than 2 years from initial diagnosis, location of relapse at an extrapulmonary site, combined local as well as systemic relapse, and high lactate dehydrogenase (LDH) levels at initial diagnosis [3-5]. No standardized treatment has been approved for relapsed ES. Local therapy at the site of relapse including radical surgery has shown to be beneficial [5]. Conventional salvage chemotherapy (CC) regimens given at relapse have led to response rates up to $29-68.1 \%$ depending on the type of regimen used and site of relapse [6-10]. The event-free survival (EFS) at 10.3 months-2 years is noted to be $22.7-26 \%$ in the literature $[8,9]$. OS rate at $1-2$ years was shown to be about $28-61 \%[7,8]$. The five-year OS was reported $20-24.5 \%$ in a retrospective study [11]. Despite its reported survival benefit, high-dose chemotherapy (HDCT) and autologous stem cell transplant (ASCT) is not routinely 
used in the United States for relapsed Ewing's sarcoma. In the last fifteen years, there have been two systematic reviews that were performed to evaluate the benefit of HDCT in Ewing's sarcoma, but these studies mainly focused on locally advanced and primary metastatic disease without major focus on relapsed Ewing's sarcoma [12, 13]. We performed a comprehensive literature search and performed systematic review to evaluate the role of HDCT along with ASCT given as an induction or consolidation regimen in patients with relapsed Ewing's sarcoma.

\section{Materials and Methods}

2.1. Source of Information and Search Strategy. Comprehensive literature search was conducted using Medline (PubMed and Ovid SP), Embase, and Cochrane Database of Systematic Review (CDSR) up to April 2017. The search strategies included various combinations of text words and controlled vocabulary when available. We used the following keywords while building search; Ewing sarcoma, relapsed/refractory, chemotherapy, high-dose chemotherapy, transplant, tandem transplant, treatment. There was no language or year limits placed on the search. Three reviewers (Pavan Tenneti, Umar Zahid, and Ahmad Iftikhar) independently applied the inclusion and exclusion criteria to the articles that were identified by the search strategy and extracted data using the standardized data extraction form. In addition, more articles were added from bibliographical data of searched articles found through search engines. Full articles of potentially useful articles were reviewed before confirming the inclusion criteria.

\subsection{Inclusion and Exclusion Criteria. We included all clinical} studies that had patients with relapsed Ewing's sarcoma who were treated with HDCT followed by ASCT using either bone marrow or peripheral blood stem cell rescue regardless of treatment settings. We excluded studies that had patients with only primary local or metastatic Ewing's sarcoma or other soft tissue sarcomas and without relapsed disease. As our article mainly aims to compare long-term outcomes of patients that got HDCT to those that did not, we also excluded ten case reports and case series that did not mention long-term outcomes of relapsed ES in their studies. Following data were extracted from the individual study: demographics, pathology, disease stage, treatment, response, and survival outcome data. When not explicitly stated, outcomes were calculated based on the information included within the published record. Outcome data were presented as mean or median, and all values are expressed to 1 decimal place unless the original article did not provide this degree of accuracy. The exceptions are $p$ values, which are expressed as reported in published record.

2.3. Outcome Measures. The primary outcome measures included complete remission (CR), partial remission (PR), progressive disease $(\mathrm{PD})$, stable disease $(\mathrm{SD})$, no response (NR), overall survival (OS), and event-free survival (EFS).

\section{Results}

3.1. Search Results. The systematic search identified a total of 1005 records. These records were screened for relevance based on their titles and abstracts. Of these studies, 64 were deemed potentially eligible and retrieved for full text review. After detailed review, a total of 43 studies were further excluded for the following reasons: duplicate study data, not focused on relapsed sarcoma, or not receiving ASCT. A total of 21 articles met our inclusion criteria. In addition, 3 published abstracts were also included (Figure 1).

3.2. Study Population. All studies included older children and younger adults with pathologically proven relapsed Ewing's sarcoma. Seven retrospective studies had patients with only relapsed Ewing's sarcoma that received HDCT $(n=205)$ (Table 1). Fourteen studies had a mixed relapsed Ewing's sarcoma, primary metastatic disease, and other soft tissue sarcomas. In these studies, a total of at least 140 patients with relapsed ES received HDCT/ASCT. Ten of these studies reported outcomes of patients with relapsed ES separately (Table 2). Four studies reported only cumulative outcome of all patients included (Table 3 ). In addition, three retrospective study abstracts consisting of patients with relapsed Ewing's sarcoma and primary metastatic disease were included (Table 4).

3.3. Retrospective Studies Containing Only Relapsed Patient Data. We identified a total of seven retrospective studies that had only relapsed ES patients who received HDCT/ASCT $(n=205)$. McTiernan et al. [15] studied 33 relapsed patients with local and metastatic disease (Table 1). All patients received CC (showed CR/PR/SD/minor response after CC) followed by HDCT/ASCT. The two- and five-year EFS rates were $43 \%$ and $39 \%$, respectively. The OS rates were $51 \%$ and $43 \%$ at two and five years, respectively. In a study performed by Ferrari et al. [17], 20 patients that showed a disease response with $\mathrm{CC}(\mathrm{CR} / \mathrm{PR})$ were given HDCT/ASCT, and the five-year OS rate was 50\% (Table 1). Similar superior results of HDCT/ASCT (given if there was disease response with CC) following CC $(n=24)$ in comparison to just CC $(n=31)$ was noted by Palmerini et al. [18]. The OS at 3 years was $33 \%$ in the former group and $22 \%$ in the later group (Table 1). The median overall survival of 49 months and median relapse-free survival of 16 months was noted in another study by Shankar et al. [19] for some patients $(n=7)$ with relapsed ES that showed response (CR/PR) with CC and subsequently received HDCT/ASCT (Table 1). Bacci et al. [3] studied 195 patients with relapsed ES, of which 35 patients received HDCT. The outcomes were reported for 33 patients in this study. The EFS at 5 years was $21.2 \%$. Among these 33 patients, thirteen of eighteen patients who had surgery/radiation prior to HDCT showed response (CR/PR) before receiving HDCT. In comparison, none of the other 15 relapsed ES patients who did not receive any prior therapy (surgery/radiation/salvage chemotherapy) showed response even after getting HDCT/ASCT. This is suggestive that HDCT might not be very effective in disease 


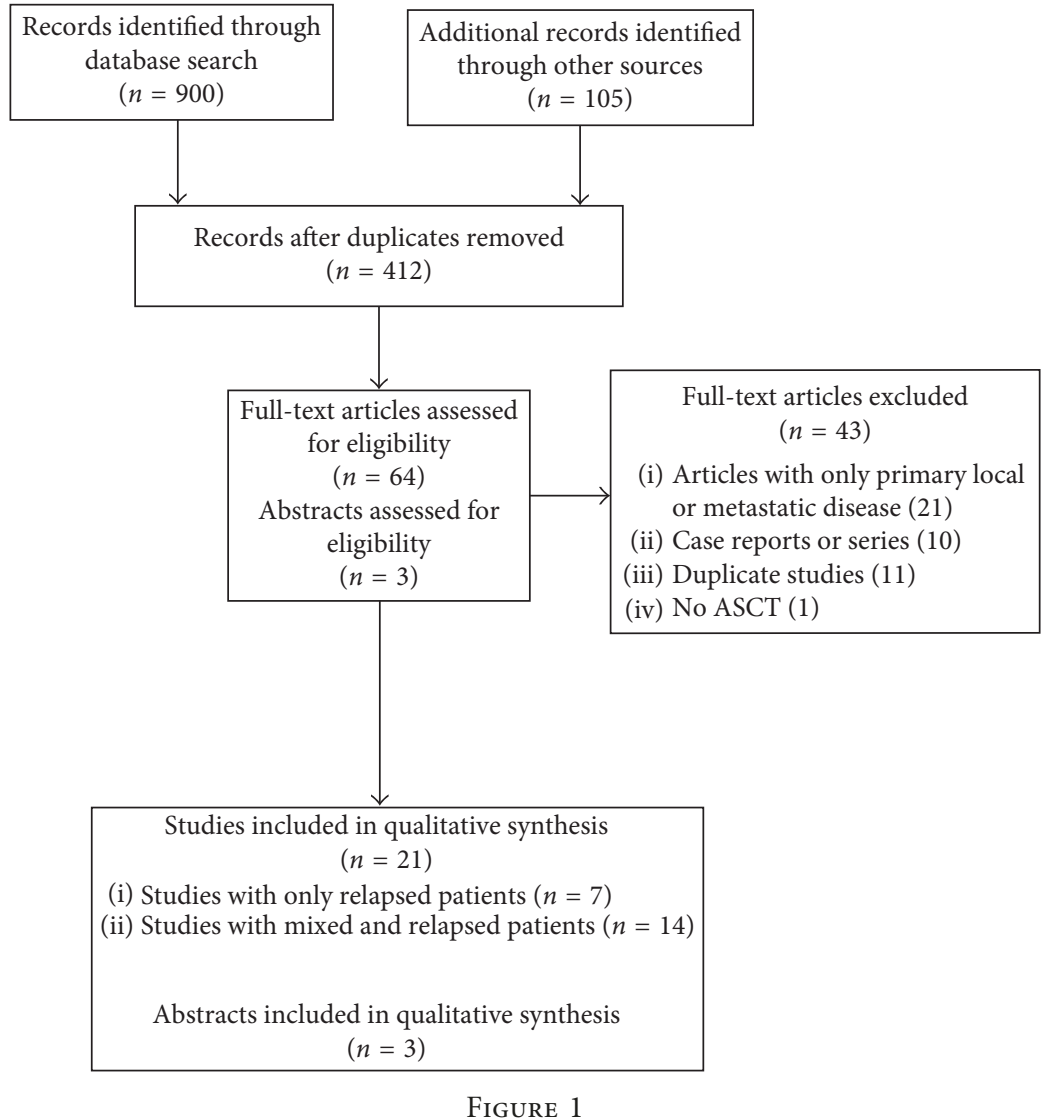

that is not responsive to first-line salvage treatment at relapse (Table 1).

Barker et al. [16] studied 55 patients with local and metastatic relapse (Table 1). All patients were treated with CC, and 27 of them showed chemosensitivity (either $\mathrm{PR} / \mathrm{CR}$ ). Thirteen of these twenty-seven patients subsequently went on to receive HDCT followed by ASCT. The five-year EFS and OS rates for this group was $61 \%$ and $77 \%$, respectively, whereas it was $21 \%$ and $22 \%$ for patients $(n=14)$ that showed response to CC but did not receive HDCT ( $p=0.018$ for both EFS and OS). Rasper et al. [14] studied 239 patients from CESS registry (cooperative Ewing's sarcoma study group) with local and metastatic relapse (Table 1). All patients received CC. In addition, 73 patients received HDCT/ASCT after CC. The outcomes were reported for 53 patients that were treated with either busulfan/melphalan or treosulfan/melphalan regimen. The outcomes were not reported for patients that were treated with consortium of other regimens $(n=20)$. The two- and five-year EFS for patients who were treated with HDCT were $44-47 \%$ and $20-24 \%$, respectively, depending on the regimen of HDCT that was used. The corresponding EFS at 2 and 5 years for patients that got only CC was $10 \%$ and $6 \%$ $(p=0.01)$. The two- and five-year OS rates for patients who got HDCT were $53-66 \%$ and $40-42 \%$, respectively, compared to $22 \%$ and $10 \%$ in patients with only CC $(p=0.01)$.

To summarize, for most studies consisting of only relapsed patients that received HDCT/ASCT after CC, EFS rates at two- and five-year ranged $42-47 \%$ and $20-61 \%$, respectively. The OS rates at two years and at three to five years ranged $50-66 \%$ and $33-77 \%$, respectively, depending on the study. Patients in Bacci et al. [3] that did not receive salvage therapy prior to HDCT did not show any clinical response (CR/PR).

3.4. Studies Containing Mixture of Relapsed and Primary Metastatic Ewing's Sarcoma Patients' Data (Results for Relapsed ES Reported Separately). There were 9 studies ( 8 retrospective and 1 prospective), in which 105 relapsed ES patients received HDCT/ASCT after CC. In most studies, only patients that showed response $(\mathrm{CR} / \mathrm{PR})$ to $\mathrm{CC}$ proceeded to get HDCT. The response status after CC was not clearly outlined in 2 studies [27, 28]. Frohlich et al. [27] studied 131 patients who were included in a registry that had 52 patients with relapsed disease. All patients received CC followed by additional HDCT/ASCT. For ES patients that relapsed within 2 years of initial disease presentation, the EFS at four years for patients that got HDCT was $17 \%$. The study compared outcomes to historical control patients where EFS at four years was $2 \%$ with only CC $(p=0.0001)$ (Table 2). Burdach et al. [22] studied 17 patients, of which 10 had relapsed ES. All patients received CC followed by HDCT/ASCT. The EFS and OS for relapsed patients at 4 years were $50 \%$ each (Table 2). Seo et al. [25] conducted a similar study on 9 patients, 5 of which had relapsed disease. At two years, EFS for relapsed patients was $40 \%$. The OS at 1 and 2 years was $60 \%$ and $40 \%$, respectively, for relapsed 


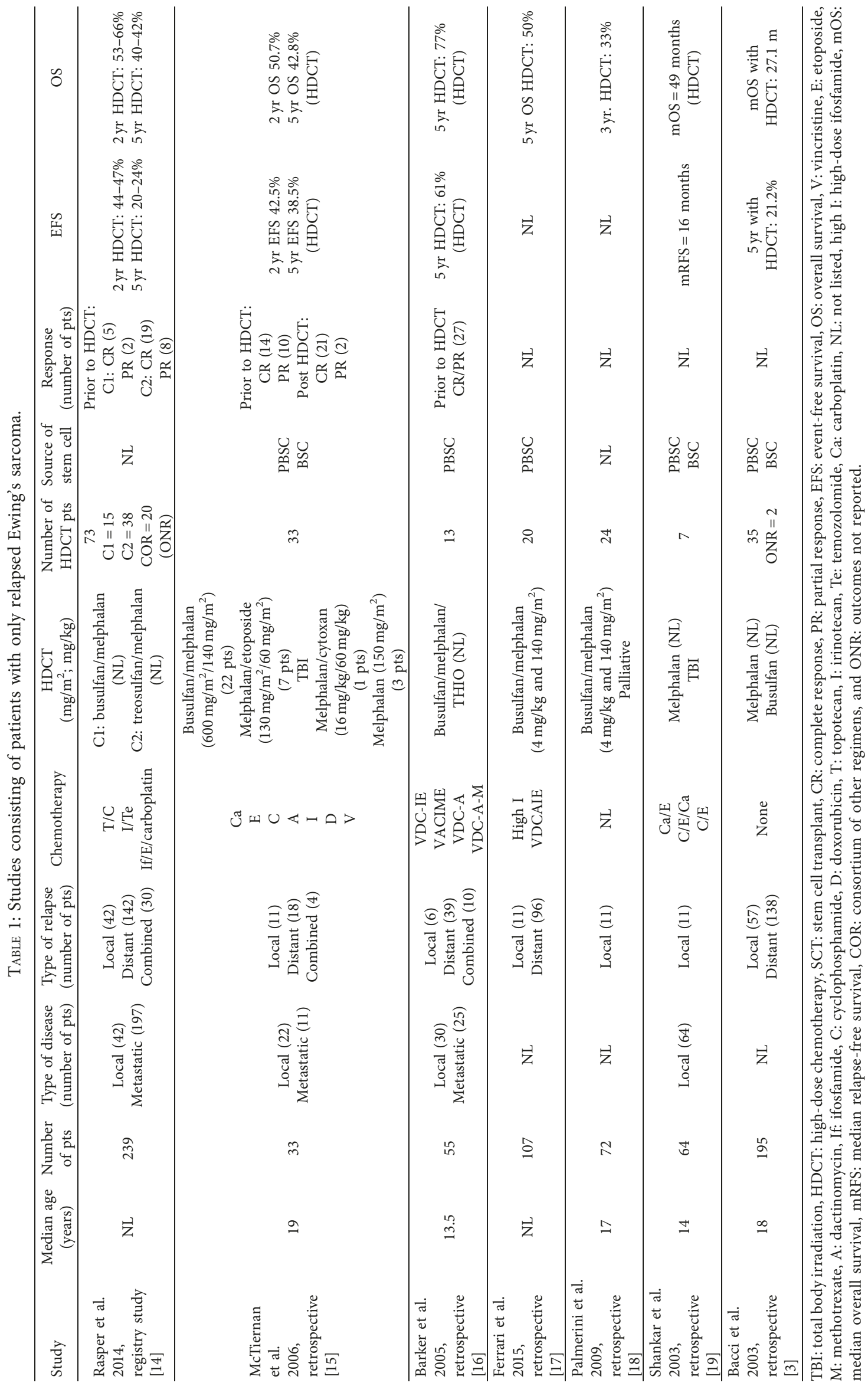




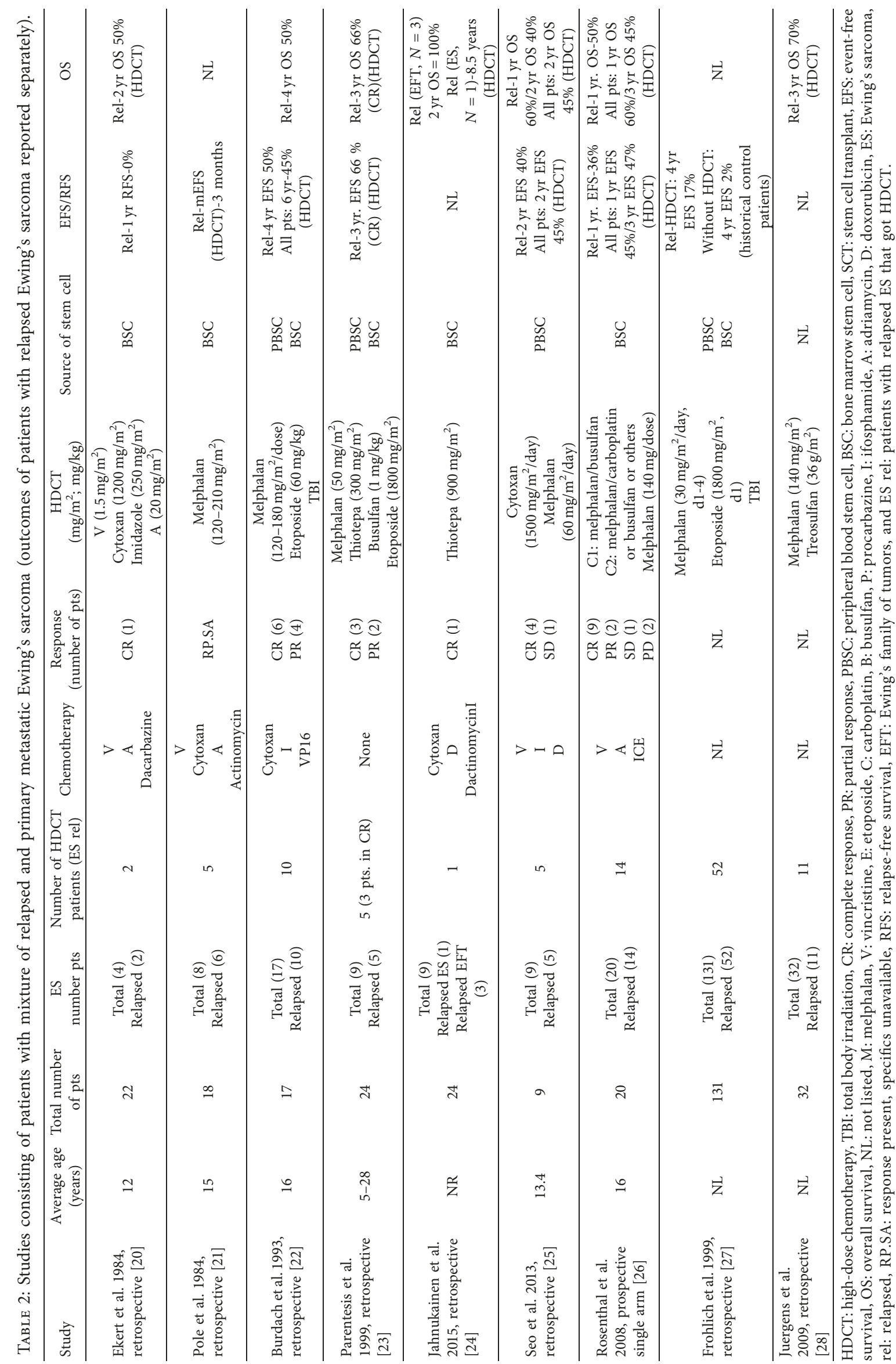




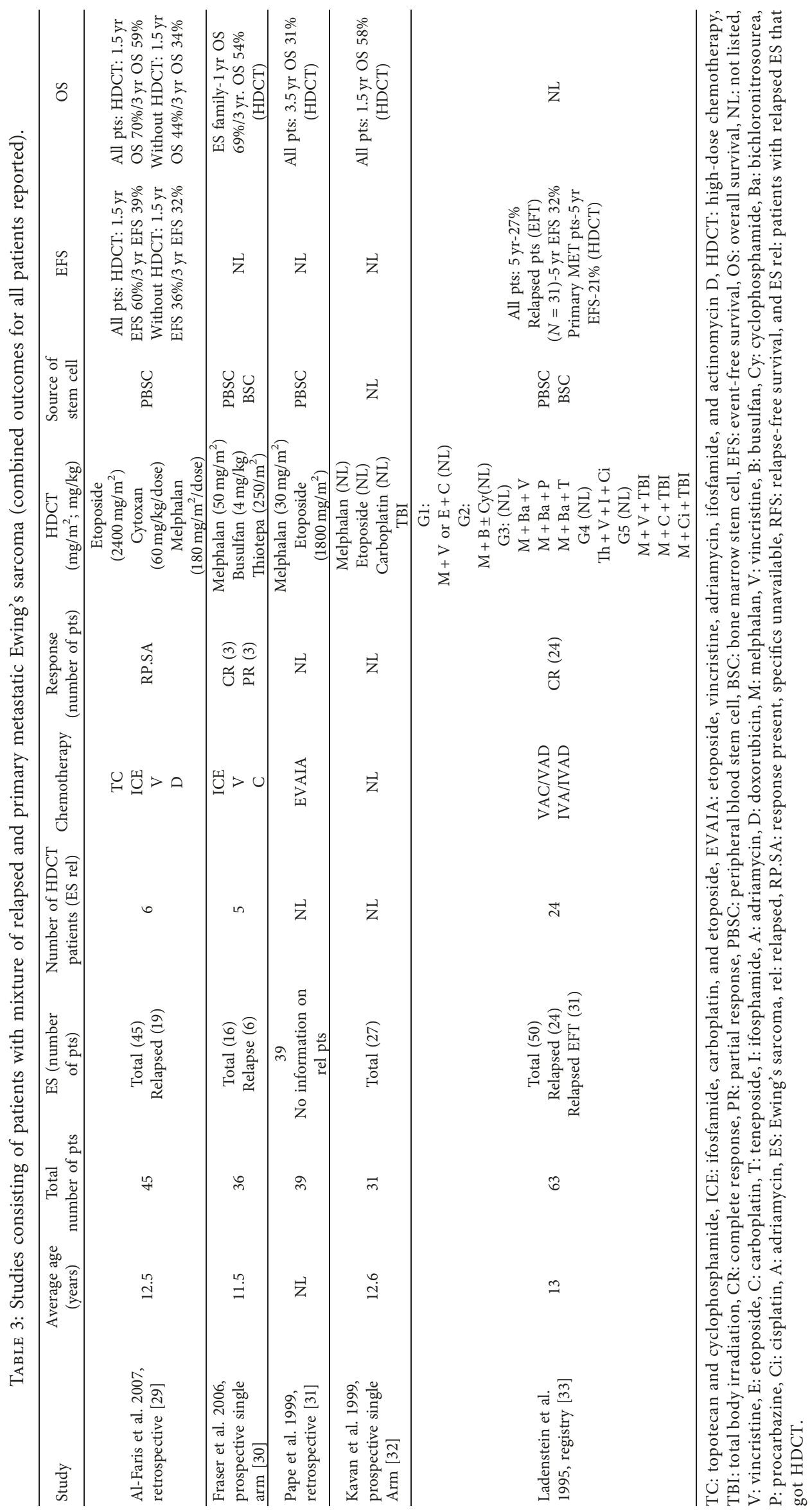




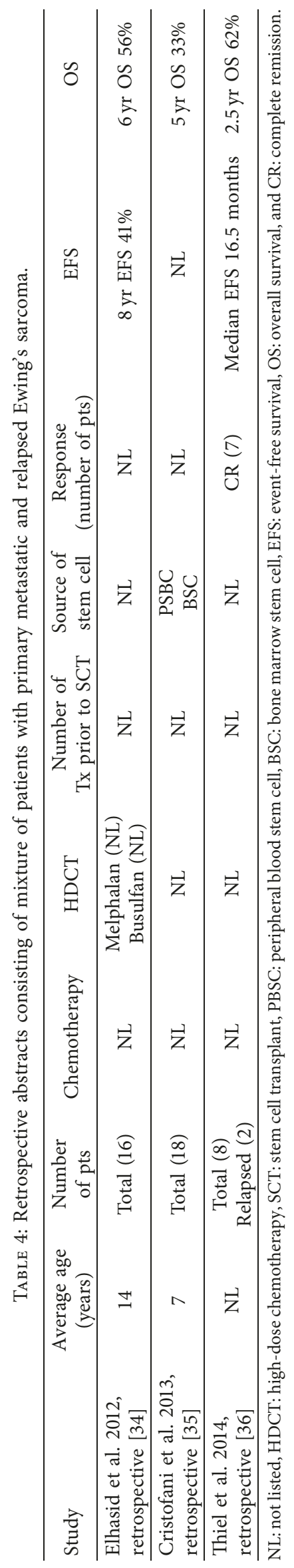


patients (Table 2). The OS was $70 \%$ reported at three years by Juergens et al. [28] in their study of 11 relapsed patients (Table 2). Similarly, Rosenthal et al. [26] studied 20 patients, 14 of whom had relapsed disease. All relapsed patients received HDCT after CC and showed EFS/OS at 1 year to be $36 \% / 50 \%$.

Ekert et al. [20] and Parentesis et al. [23] reported results on smaller group of relapsed patients. The OS at 2 years for the 2 relapsed patients in the former study was $50 \%$ (CC followed by HDCT), and it was $66 \%$ at three years for 3 relapsed patients who were in CR (after receiving CC) prior to HDCT/ASCT in the later study. Jahnukainen et al. [24] noted that the only relapsed ES patient in their study who received thiotepa-based HDCT following CC survived for 8.5 years. In the same study, the cumulative OS for 3 patients with relapsed Ewing's family of tumors (primitive neuroectodermal tumors and Ewing's sarcoma) at 2 years was $100 \%$ (Table 2). Graham-Pole et al. [21] studied 8 patients, 6 of which had relapsed disease. In this study, HDCT was given as induction regimen. None of the relapsed ES patients survived beyond 3 months. This study was performed in 1984 when transplant-related mortality was high, and patients received HDCT as an induction regimen without prior CC; these factors would have contributed to poor outcomes (Table 2).

3.5. Studies Containing Mixture of Relapsed and Primary Metastatic Ewing's Sarcoma Patients' Data (Results for Relapsed ES Not Reported Separately). Five studies (2 prospective and 3 retrospective) reported combined outcomes of all patients in their study. At least 35 relapsed ES patients received HDCT. In most studies, patients who proceeded to get HDCT/ASCT showed initial response (CR/PR) with CC. The response status of patients was not clearly outlined in one study [31]. Al-Faris et al. [29] studied 45 patients, out of which 19 had relapsed disease. All patients received CC. Twenty patients received additional HDCT/ASCT (this included 6 patients with relapsed ES). The one-and-half-year EFS and three-year EFS for patients who received HDCT were 60 and 39\%, respectively, whereas for patients who got only CC, EFS was 36 and $32 \%(p=0.08)$, respectively. The one and half and three-year OS for patients who received HDCT was $70 \%$ and 59\%, respectively, whereas it was 44 and $34 \%$ for patients who got only CC $(p=0.06)$ (Table 3$)$. Pape et al. [31] presented results of 39 patients that were mix of primary metastatic and relapsed ES. All patients received CC followed by HDCT/ASCT. OS at three and half years was 31 percent for the whole group (Table 3 ). In another study by Fraser et al. [30] that consisted of sixteen patients, six had relapsed disease. Patients got CC followed by HDCT and ASCT. For patients with ESW (ES and desmoplastic small round cell tumors) at one and three years, OS was 69 and 54 percent, respectively (Table 3). Ladenstein et al. [33] performed a registry study consisting of 31 patients with relapsed EFT (Ewing sarcoma family of tumors) consisting of patients with Ewing's sarcoma and primitive neuroectodermal tumors. Twenty-four of these had relapsed ES. All patients received CC followed by HDCT and ASCT.
EFS for all relapsed patients at five years was $32 \%$. In addition, the cumulative outcome for all patients $(n=63)$ included in the study (primary metastatic EFT and relapsed EFT) at 5 years was 27\% (Table 3). Kavan et al. [32] reported one and half year OS to be $58 \%$ for the 31 patients (mix of advanced primary ES/relapsed ES) studied who received CC followed by HDCT and ASCT (Table 3).

To summarize, for most studies that only reported combined outcomes for patients with relapsed/primary metastatic ES along with other malignancies (that got HDCT/ASCT following CC), EFS at 1.5-3 and 5 years was $39-60 \%$ and $27 \%$, respectively. OS at $1-1.5$ and $3-3.5$ years was $58-70 \%$ and $31-59 \%$, respectively. In studies that reported outcomes for relapsed ES patients separately (that received $\mathrm{HDCT} / \mathrm{ASCT}$ after CC), EFS at 1-3 and 4 years was $36-66 \%$ and $17-50 \%$, respectively. The OS at $1-2$ and 3-4 years was $40-60 \%$ and $50-70 \%$, respectively, depending on the study. Patients in Graham-Pole et al. [21] did exceptionally poor possibly because of lack of usage of CC prior to HDCT. The three patients with relapsed EFT (including one patient with relapsed ES) along with patients with advanced primary ES in Jahnukainen et al. [24] did exceptionally well on thiotepa HDCT regimen, the reasons not being very clear.

3.6. Retrospective Studies (Abstracts) Containing Mixture of Relapsed and Primary Metastatic Ewing's Sarcoma Patients' Data. We also found three abstracts $(n=42)$ that were published by Thiel et al. [36], Cristofani et al. [35], and Elhasid et al. [34]. All studies had a mixed group of patients consisting relapsed and primary metastatic disease. The results were also reported for combined group and not separately for relapsed patients. To summarize, the OS at three to six years ranged from $33-62 \%$ (Table 4).

3.7. Comparative Studies of Relapsed Ewing's Sarcoma. Five studies compared results of relapsed patients who received HDCT along with ASCT after CC to those who received only CC $[3,14,16-18]$ The EFS at 2 and 5 years for patients that got HDCT/ASCT after CC was $44-47 \%$ and $20-61 \%$, whereas it was $10 \%$ and $0-7 \%$, respectively, for patients who got only CC. The OS at $2-5$ years was $33-77 \%$ for HDCT/ASCT (after CC), whereas it was 5-22\% for only CC. In addition, Bacci et al. [3] reported mOS with HDCT/ASCT (after surgery/radiation) to be 23 months compared to 11.1 months with only CC. Two studies demonstrated superior results of HDCT/ASCT in chemosensitive relapsed ES (after CC) through direct comparison of survival outcomes of patients that got HDCT (after CC) to those that did not receive it $[14,16]$. They reported EFS and OS after HDCT at $2-5$ years to be $22-61 \%$ and $41-77 \%$. The EFS and OS with just CC (without additional HDCT) at 2-5 years were $18-31 \%$ and $22-45 \%$, respectively $[14,16]$. These comparative studies clearly show that HDCT/ASCT given as consolidative measure after initial salvage regimen clearly has a survival benefit (Table 5). 


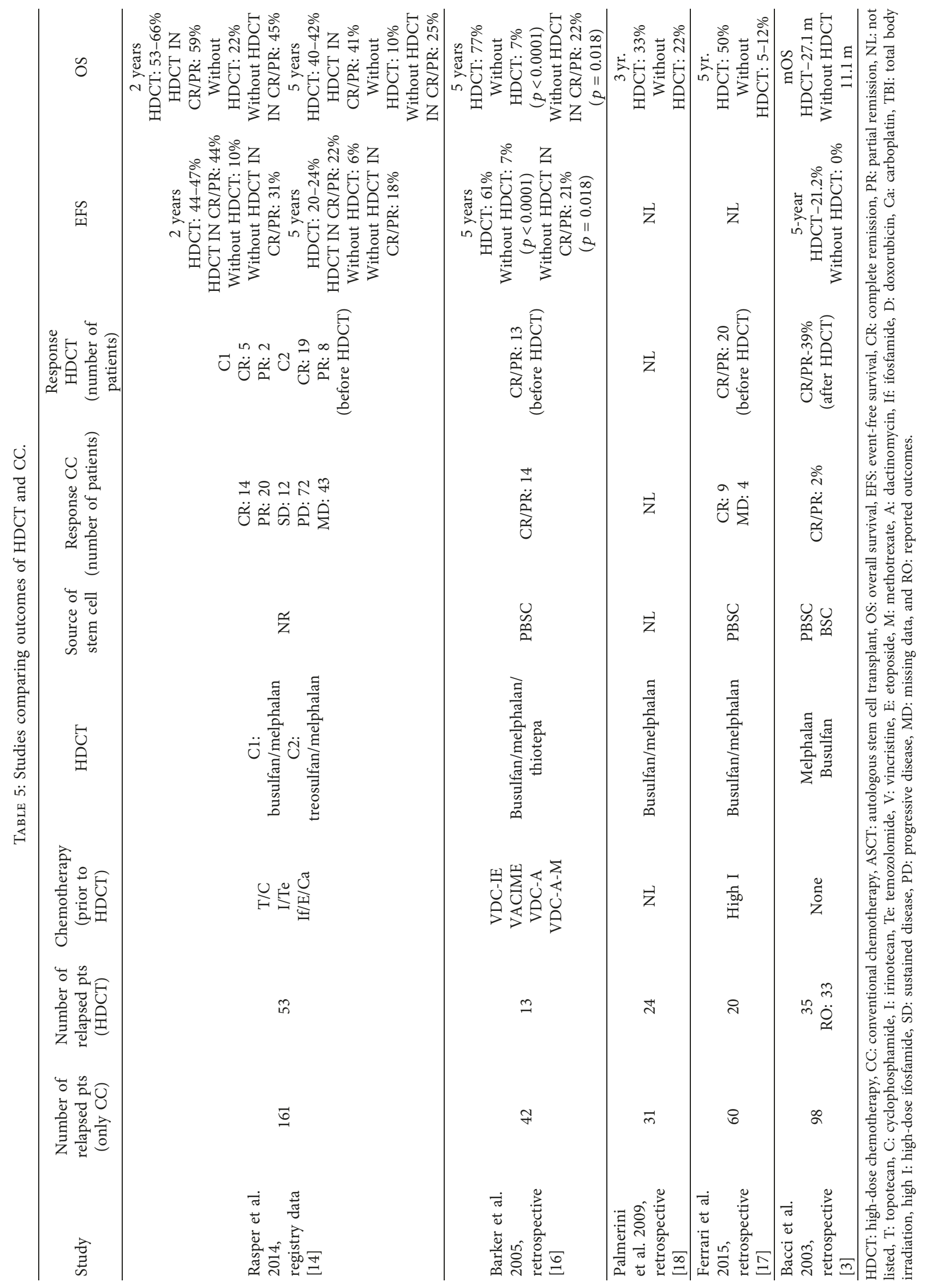




\section{Discussion}

In patients with relapsed Ewing's sarcoma, there is not one established regimen as the standard of care. Regarding conventional therapy salvage options, many regimens have been tried with variable results. A phase II study with ifosfamide with mesna along with etoposide showed that sixteen of seventeen patients with relapsed Ewing's sarcoma showed responses (either CR/PR). The patients in this study were only followed for 10 weeks; hence, long-term survival is not known [37]. Another phase II study using ICE regimen (ifosfamide, carboplatin, and etoposide) showed response rate of $51 \%$ and 1 -year and 2 -year OS rates of $49 \%$ and $28 \%$, respectively [7]. Among nonifosfamide regimens, docetaxel along with gemcitabine showed overall response rate (ORR) of $29 \%$ and median duration of response of 4.8 months [6]. Cyclophosphamide and topotecan showed response rate of $44 \%$ and 2 -year EFS rate of $26 \%$ in study with 54 relapsed Ewing's sarcoma patients [8]. Irinotecan and temozolomide resulted in response rate of $63 \%$ and time to progression of eight months [10]. A study with patients receiving VIT regimen (vincristine, irinotecan, and temozolomide) showed ORR of $68 \%$ with $22.7 \%$ patients alive and with no evidence of disease at 10.3 months [9]. In another retrospective study conducted on 107 patients with relapsed Ewing's sarcoma with either etoposide and cisplatin or etoposide and carboplatin showed EFS of 6.5 and 14 months, respectively, with 5 -year OS rates of $20 \%$ and $24.5 \%$, respectively [11]. Collectively, these data suggest the need for further improvement in treatment outcomes in Ewing's sarcoma patients.

The usage of HDCT followed by ASCT rescue is based on observation that outcome in many malignancies that are chemosensitive is dependent on the dosage of chemotherapy used. Steep dose-response is noticed for both toxic and therapeutic effects. Preclinical studies documented linear log correlation between dose and tumor cytotoxicity. An increase in dose by three to tenfold, particularly for alkylating agents, can result in a multiple log increase in tumor cell death. Myelosuppression is one of the major side effects at this dose [38]. ASCT helps to rescue marrow and allows for further dose escalation [39]. HDCT along with ASCT has shown to benefit in the treatment of various malignancies including high-grade germ cell tumors [40, 41], newly diagnosed multiple myeloma [42, 43], and relapsed Hodgkin's and non-Hodgkin lymphomas [44]. HDCT with ASCT has also shown benefits in improving survival in chemosensitive soft tissue sarcomas including relapsed osteosarcoma patients $[45,46]$. HDCT/ASCT has shown benefit in improving OS and EFS for relapsed Ewing's sarcoma in single institute studies $[15,16,22]$.

We compared outcomes of HDCT with ASCT given as consolidation regimen after CC to results from historical studies which used only CC. In most studies, patients with relapsed ES that received HDCT/ASCT showed initial chemosensitivity (CR/PR) to CC. The OS was $20-60 \%$ at $1-2$ years and $20-25 \%$ at 5 years when only CC was used in historical studies $[7,8,11]$. The EFS at one to two years was around $25 \%[8,9]$. For most studies utilizing HDCT/ASCT that presented outcomes for only relapsed patients, the EFS at two and five years was $42-47 \%$ and $20-61 \%$, respectively. The OS for this group at 2 and 3-5 years ranged from 50 to $66 \%$ and 33-77\%, respectively. For studies utilizing HDCT/ASCT that consisted data on mixed patients (relapsed, primary metastatic disease, and other soft tissue sarcomas) which reported outcomes for relapsed ES separately, the cumulative EFS at $1-3$ and 4 years was $36-66 \%$ and $17-50 \%$, respectively. The OS at $1-2$ and 3-4 years was $40-60 \%$ and $50-70 \%$. The other studies consisting of mixed patients reported outcomes for the whole group. The EFS at $1.5-3$ and 5 years was $39-60 \%$ and $27 \%$, respectively. The OS from the same group at $1-1.5$ and $3-3.5$ years was $58-70 \%$ and $31-59 \%$, respectively. For data presented in the abstract format, the cumulative OS (not separated for relapsed ES) at three to six years was 30-60\% [34-36, 47]. As evident, the OS and EFS with HDCT followed by ASCT given after CC was better than the outcomes from historical studies where only CC was used.

Five studies that compared outcomes of patients with relapsed ES who received HDCT and ASCT after CC with those who received only CC showed improved survival with HDCT/ASCT [3, 14, 16-18]. Patients in two studies though did not show improved outcomes with HDCT. Patients in the work of Pole et al. [21] did not receive CC prior to HDCT, which might have contributed to poor outcome. This is also the possible contributing cause for poor outcomes for a subgroup of 15 patients (did not get surgery/radiation prior to HDCT) that did not show response (CR/PR) even after getting HDCT/ASCT in a study done by Bacci et al. [3] Results of both these studies suggest that HDCT probably is not very effective in disease that is not responsive to initial salvage therapy (chemotherapy or other treatments) at relapse.

Among all positive OS survival outcomes, HDCT regimens used in eight studies (Barker et al. [16], Ferrari et al. [48], Juergens et al. [28], Burdach et al. [22], Elhasid et al. [34], Al-Faris et al. [29], Jahnukainen et al. [24], and Parenthesis et al. [23]), seemed to have resulted in best OS among others. The HDCT regimen used in these seven studies are listed in Tables 1-3. Most regimens contained melphalan in combination with other cytotoxic agents. Given the heterogeneity among studies which used HDCT, it is difficult to determine if there was a superior regimen with better outcomes when compared to others. With realization of limited data, all three patients with relapsed EFT in Jahnukainen et al. [24] study received thiotepa-based HDCT and did exceptionally well and were surviving at the end of two and half years.

The superior outcomes seen in the studies could also have been impacted by many factors, such as selection bias in favor of younger, fit patients who were offered HDCT. Most studies included in our analysis were retrospective in nature, and patients who did not respond to CC did not proceed to receive $\mathrm{HDCT}$, thus adding a further selection bias for chemosensitive patients, who are more likely to be offered HDCT/ASCT, which may affect the positive impact observed with this intervention. Limitation of our review also includes significant heterogeneity in included study designs, 
type of CC and HDCT used, local versus metastatic relapse, different inclusion criteria, follow-up duration, and study end points in various trials included in our analysis. These factors made it difficult to perform meta-analysis of the available data. In addition, patients included in studies had combined data on relapsed and primary metastatic disease. Despite these limitations, results of this review suggest that HDCT has a positive impact on survival.

\section{Conclusion}

In conclusion, HDCT and ASCT given as a consolidation treatment after CC appear to be beneficial in improving survival in patients with relapsed Ewing's sarcoma. It appears to be more prominent in chemosensitive diseases. HDCT given as induction treatment did not appear to be beneficial in improving survival, but it was used in only two studies. The heterogeneity in the included studies suggests that prospective randomized controlled studies are needed to definitively show the benefit for single and two cycles of HDCT and ASCT in relapsed Ewing's sarcoma.

\section{Conflicts of Interest}

The authors declare that there are no conflicts of interest.

\section{Authors' Contributions}

Pavan Tenneti, Umar Zahid, and Faiz Anwer designed the study. Pavan Tenneti, Umar Zahid, Ahmad Iftikhar, and Faiz Anwer searched for relevant studies for the systematic review. All authors performed the study, contributed to data extraction, analyzed the data, and wrote the paper.

\section{Acknowledgments}

This work was supported by Grant P30 CA023074 from the National Cancer Institute, National Institutes of Health, Bethesda, MD.

\section{References}

[1] M. Paulussen, S. Bielack, H. Jurgens et al., "Ewing's sarcoma of the bone: ESMO clinical recommendations for diagnosis, treatment and follow-up," Annals of Oncology, vol. 20, no. 4, pp. 140-142, 2009.

[2] M. Stahl, A. Ranft, M. Paulussen et al., "Risk of recurrence and survival after relapse in patients with Ewing sarcoma," $P e$ diatric Blood and Cancer, vol. 57, no. 4, pp. 549-553, 2011.

[3] G. Bacci, S. Ferrari, A. Longhi et al., "Therapy and survival after recurrence of Ewing's tumors: the Rizzoli experience in 195 patients treated with adjuvant and neoadjuvant chemotherapy from 1979 to 1997," Annals of Oncology, vol. 14, no. 11, pp. 1654-1659, 2003.

[4] P. J. Leavey, L. Mascarenhas, N. Marina et al., "Prognostic factors for patients with Ewing sarcoma (EWS) at first recurrence following multi-modality therapy: a report from the Children's Oncology Group," Pediatric Blood and Cancer, vol. 51, no. 3, pp. 334-338, 2008.

[5] C. Rodriguez-Galindo, C. A. Billups, L. E. Kun et al., "Survival after recurrence of Ewing tumors: the St Jude Children's
Research Hospital experience, 1979-1999," Cancer, vol. 94, no. 2, pp. 561-569, 2002.

[6] F. Navid, J. R. Willert, M. B. McCarville et al., "Combination of gemcitabine and docetaxel in the treatment of children and young adults with refractory bone sarcoma," Cancer, vol. 113, no. 2, pp. 419-425, 2008.

[7] P. Van Winkle, A. Angiolillo, M. Krailo et al., "Ifosfamide, carboplatin, and etoposide (ICE) reinduction chemotherapy in a large cohort of children and adolescents with recurrent/ refractory sarcoma: the Children's Cancer Group (CCG) experience," Pediatric Blood and Cancer, vol. 44, no. 4, pp. 338-347, 2005.

[8] A. Hunold, N. Weddeling, M. Paulussen et al., "Topotecan and cyclophosphamide in patients with refractory or relapsed Ewing tumors," Pediatric Blood and Cancer, vol. 47, no. 6, pp. 795-800, 2006.

[9] A. Raciborska, K. Bilska, K. Drabko et al., "Vincristine, irinotecan, and temozolomide in patients with relapsed and refractory Ewing sarcoma," Pediatric Blood and Cancer, vol. 60, no. 10, pp. 1621-1625, 2013.

[10] D. A. Casey, L. H. Wexler, M. S. Merchant et al., "Irinotecan and temozolomide for Ewing sarcoma: the Memorial SloanKettering experience," Pediatric Blood and Cancer, vol. 53, no. 6, pp. 1029-1034, 2009.

[11] A. M. van Maldegem, C. Benson, P. Rutkowski et al., "Etoposide and carbo-or cisplatin combination therapy in refractory or relapsed Ewing sarcoma: a large retrospective study," Pediatric Blood and Cancer, vol. 62, no. 1, pp. 40-44, 2015.

[12] J. Rosenthal and A. B. Pawlowska, "High-dose chemotherapy and stem cell rescue for high-risk Ewing's family of tumors," Expert Review of Anticancer Therapy, vol. 11, no. 2, pp. 251262, 2011.

[13] S. A. Kalambakas, T. B. Moore, and S. A. Feig, "Megatherapy and stem cell transplantation for Ewing's family of tumors: a critical review of current literature," Pediatric Transplantation, vol. 8, no. 5, pp. 83-88, 2004.

[14] M. Rasper, S. Jabar, A. Ranft, H. Jürgens, S. Amler, and U. Dirksen, "The value of high-dose chemotherapy in patients with first relapsed Ewing sarcoma," Pediatric Blood and Cancer, vol. 61, no. 8, pp. 1382-1386, 2014.

[15] A. McTiernan, D. Driver, M. P. Michelagnoli, A. M. Kilby, and J. S. Whelan, "High dose chemotherapy with bone marrow or peripheral stem cell rescue is an effective treatment option for patients with relapsed or progressive Ewing's sarcoma family of tumours," Annals of Oncology, vol. 17, no. 8, pp. 1301-1305, 2006.

[16] L. M. Barker, T. W. Pendergrass, J. E. Sanders, and D. S. Hawkins, "Survival after recurrence of Ewing's sarcoma family of tumors," Journal of Clinical Oncology, vol. 23, no. 19, pp. 4354-4362, 2005.

[17] S. Ferrari, R. Luksch, K. Sundby Hall et al., "Post-relapse survival in patients with Ewing sarcoma," Pediatric Blood Cancer, vol. 62, no. 6, pp. 994-999, 2015.

[18] E. Palmerini, M. Colangeli, C. Nanni et al., "High-dose chemotherapy with autologous stem cell transplantation for relapsed Ewing's sarcoma," Journal of Clinical Oncology, vol. 27, no. 15, p. 10545, 2009.

[19] A. G. Shankar, S. Ashley, A. W. Craft, and C. R. Pinkerton, "Outcome after relapse in an unselected cohort of children and adolescents with Ewing sarcoma," Medical and Pediatric Oncology, vol. 40, no. 3, pp. 141-147, 2003.

[20] H. Ekert, K. Tiedemann, K. D. Waters, and W. M. Ellis, "Experience with high dose multiagent chemotherapy and 
autologous bone marrow rescue in the treatment of twentytwo children with advanced tumours," Australian Paediatric Journal, vol. 20, no. 3, pp. 195-201, 1984.

[21] J. Graham-Pole, H. M. Lazarus, R. H. Herzig et al., "High-dose melphalan therapy for the treatment of children with refractory neuroblastoma and Ewing's sarcoma," American Journal of Pediatric Hematology/Oncology, vol. 6, no. 1, pp. 17-26, 1984.

[22] S. Burdach, H. Jürgens, C. Peters et al., "Myeloablative radiochemotherapy and hematopoietic stem-cell rescue in poor-prognosis Ewing's sarcoma," Journal of Clinical Oncology, vol. 11, no. 8, pp. 1482-1488, 1993.

[23] J. Perentesis, E. Katsanis, T. E. DeFor, J. P. Neglia, and N. K. C. Ramsay, "Autologous stem cell transplantation for high-risk pediatric solid tumors," Bone Marrow Transplantation, vol. 24, no. 6, pp. 609-615, 1999.

[24] K. Jahnukainen, P. Kallio, A. Koivusalo, and U. M. SaarinenPihkala, "High-dose thiotepa as consolidation therapy with autologous hematopoietic stem cell transplantation for highrisk Ewing family tumors: Single-institution experience," Journal of Pediatric Hematology/Oncology, vol. 37, no. 7, pp. 536-542, 2015.

[25] J. Seo, D. H. Kim, J. S. Lim et al., "High-dose chemotherapy and autologous peripheral blood stem cell transplantation in the treatment of children and adolescents with ewing sarcoma family of tumors," Korean Journal of Pediatrics, vol. 56, no. 9, pp. 401-406, 2013.

[26] J. Rosenthal, E. Bolotin, M. Shakhnovits et al., "High-dose therapy with hematopoietic stem cell rescue in patients with poor prognosis Ewing family tumors," Bone Marrow Transplantation, vol. 42, no. 5, pp. 311-318, 2008.

[27] B. Frohlich, S. Ahrens, S. Burdach et al., "High-dosage chemotherapy in primary metastasized and relapsed Ewing's sarcoma," Klinische Pädiatrie, vol. 211, no. 4, pp. 284-290, 1999.

[28] H. Juergens, A. Ranft, M. Paulussen et al., "Treosulfan-based high-dose chemotherapy with autologous stem cell transplantation in high-risk Ewing sarcoma," Journal of Clinical Oncology, vol. 27, no. 15, p. 10546, 2009.

[29] N. Al-Faris, T. Al Harbi, C. Goia, A. Pappo, J. Doyle, and A. Gassas, "Does consolidation with autologous stem cell transplantation improve the outcome of children with metastatic or relapsed Ewing sarcoma?," Pediatric Blood and Cancer, vol. 49, no. 2, pp. 190-195, 2007.

[30] C. J. Fraser, B. J. Weigel, J. P. Perentesis et al., “Autologous stem cell transplantation for high-risk Ewing's sarcoma and other pediatric solid tumors," Bone Marrow Transplantation, vol. 37, no. 2, pp. 175-181, 2006.

[31] H. Pape, H.-J. Laws, S. Burdach et al., "Radiotherapy and highdose chemotherapy in advanced Ewing's tumors," Strahlentherapie und Onkologie, vol. 175, no. 10, pp. 484-487, 1999.

[32] P. Kavan, J. Novotny, E. Kabickova et al., "High dose chemotherapy in children with Ewing sarcoma and peripheral neuroectodermal tumor," Klinicka Onkologie, vol. 12, pp. 120-123, 1999.

[33] R. Ladenstein, T. Philip, C. Lasset et al., "Impact of megatherapy in children with high-risk Ewing's tumours in complete remission: a report from the EBMT Solid Tumour Registry," Bone Marrow Transplant, vol. 15, no. 5, pp. 697705, 1995.

[34] R. Elhasid, M. W. B. Arush, S. Postovsky et al., "Autologous stem cell transplantation in Ewing's sarcoma: the experience of Rambam medical centre and Tel Aviv medical centre," Bone Marrow Transplantation, vol. 47, pp. S425-S426, 2012.
[35] L. M. Cristofani, M. T. Almeida, and J. Fernandes, "Megachemotherapy with hematopoietic stem cell rescue for the treatment of children with advanced solid tumors," Pediatric Transplantation, vol. 17, no. 2, p. 192, 2013.

[36] U. Thiel, A. Wawer, I. Teichert et al., "Stem cell rescue from irradiation of multiple tumor sites combined with high-dose chemotherapy, followed by reduced intensity conditioning and haplodisparate stem cell transplantation in patients with advanced pediatric sarcomas: preliminary results," Bone Marrow Transplantation, vol. 49, p. S207, 2014.

[37] J. S. Miser and J. E. Sanders, "High-dose chemotherapy and systemic adjuvant irradiation in Ewing's sarcoma," Bone Marrow Transplantation, vol. 14, no. 1, p. S54, 1994.

[38] E. Frei and G. P. Canellos, "Dose: a critical factor in cancer chemotherapy," American Journal of Medicine, vol. 69, no. 4, pp. 585-594, 1980.

[39] R. C. Seeger and C. P. Reynolds, "Treatment of highrisk solid tumors of childhood with intensive therapy and autologous bone marrow transplantation," Pediatric Clinics of North America, vol. 38, no. 2, pp. 393-424, 1991.

[40] U. De Giorgi, S. Richard, M. Badoglio et al., "Salvage highdose chemotherapy in female patients with relapsed/ refractory germ cell tumors: a retrospective analysis of the European Group for Blood and Marrow Transplantation (EBMT)," Annals of Oncology, vol. 28, no. 8, pp. 1910-1916, 2017.

[41] F. Petrelli, A. Coinu, G. Rosti, P. Pedrazzoli, and S. Barni, "Salvage treatment for testicular cancer with standard- or high-dose chemotherapy: a systematic review of 59 studies," Medical Oncology, vol. 34, no. 8, p. 133, 2017.

[42] J. A. Child, G. J. Morgan, F. E. Davies et al., "High-dose chemotherapy with hematopoietic stem-cell rescue for multiple myeloma," New England Journal of Medicine, vol. 348, no. 19, pp. 1875-1883, 2003.

[43] B. Barlogie, M. Attal, J. Crowley et al., "Long-term follow-up of autotransplantation trials for multiple myeloma: update of protocols conducted by the intergroupe francophone du myelome, southwest oncology group, and university of arkansas for medical sciences," Journal of Clinical Oncology, vol. 28, no. 7, pp. 1209-1214, 2010.

[44] T. Philip, C. Guglielmi, A. Hagenbeek et al., "Autologous bone marrow transplantation as compared with salvage chemotherapy in relapses of chemotherapy-sensitive non-Hodgkin's lymphoma," New England Journal of Medicine, vol. 333, no. 23, pp. 1540-1545, 1995.

[45] A. Lashkari, W. A. Chow, F. Valdes et al., "Tandem high-dose chemotherapy followed by autologous transplantation in patients with locally advanced or metastatic sarcoma," Anticancer Research, vol. 29, no. 8, pp. 3281-3288, 2009.

[46] F. Fagioli, M. Aglietta, A. Tienghi et al., "High-dose chemotherapy in the treatment of relapsed osteosarcoma: an Italian sarcoma group study," Journal of Clinical Oncology, vol. 20, no. 8, pp. 2150-2156, 2002.

[47] U. Thiel, M. Schöniger, R. Haase et al., "Stem cell rescue after irradiation of multiple tumor sites combined with high-dose chemotherapy-high long-term survival in patients with advanced translocation positive pediatric sarcomas without bone marrow involvement," Oncology Research and Treatment, vol. 39, p. 39, 2016.

[48] S. Ferrari, K. Sundby Hall, R. Luksch et al., "Nonmetastatic Ewing family tumors: high-dose chemotherapy with stem cell rescue in poor responder patients. results of the Italian Sarcoma Group/Scandinavian Sarcoma Group III protocol," Annals of Oncology, vol. 22, no. 5, pp. 1221-1227, 2011. 


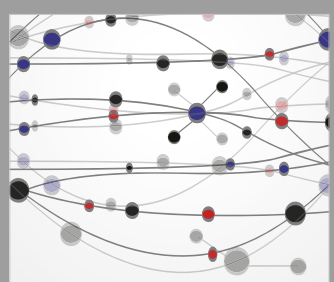

The Scientific World Journal
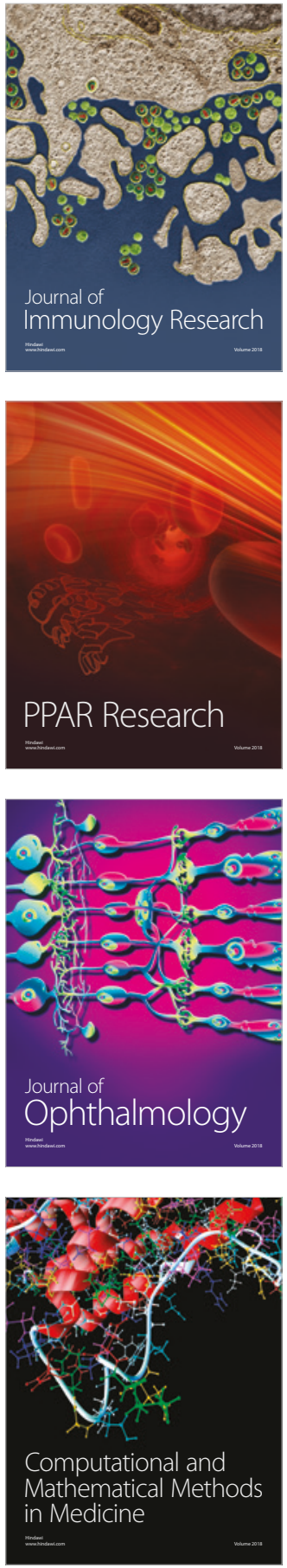

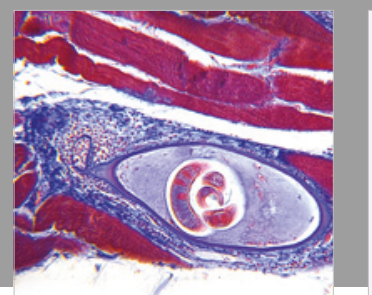

Gastroenterology Research and Practice

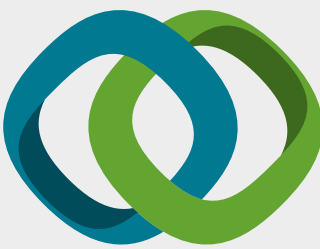

\section{Hindawi}

Submit your manuscripts at

www.hindawi.com
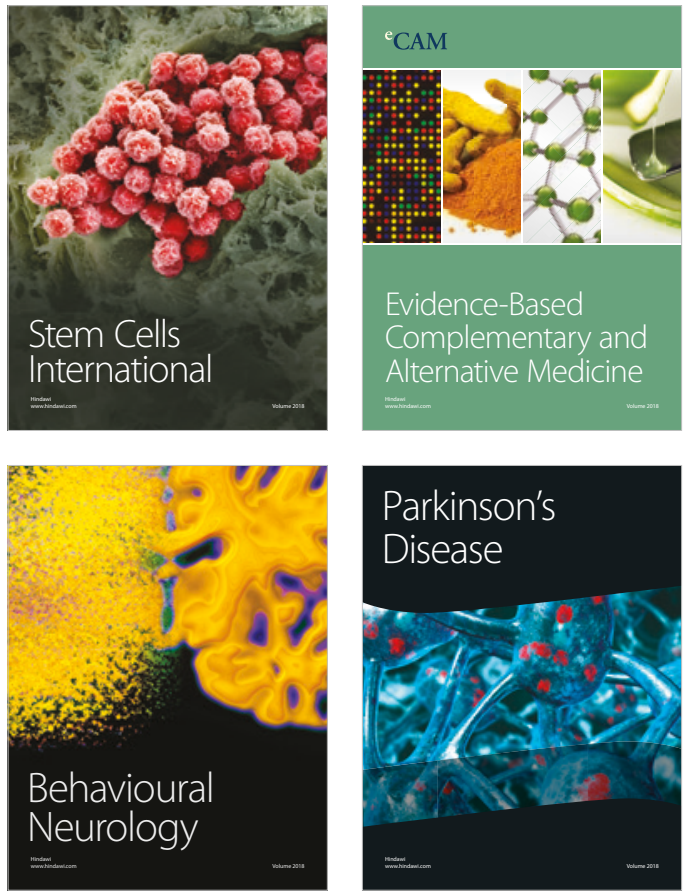

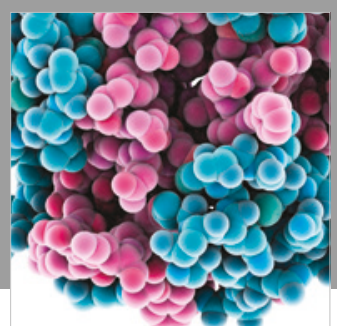

ournal of

Diabetes Research

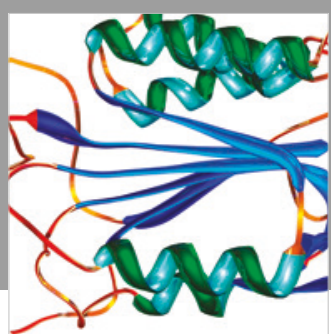

Disease Markers
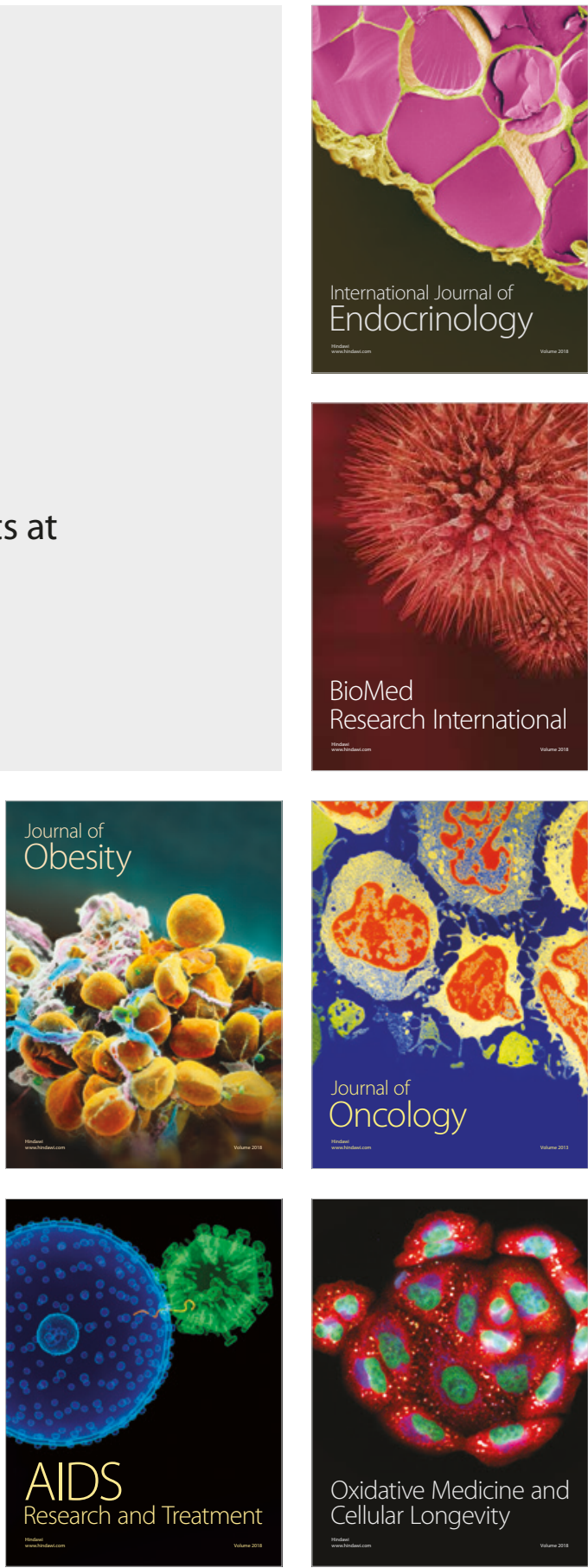